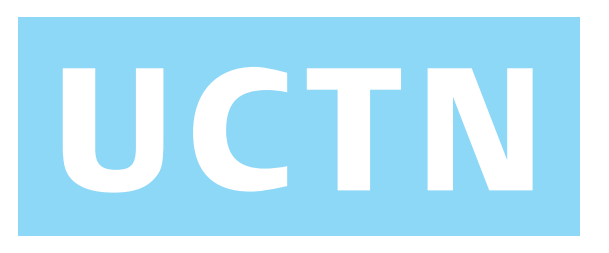

\title{
Incorrect Macroscopic Diagnosis of Colonic Carcinoma Made at Endoscopy
}

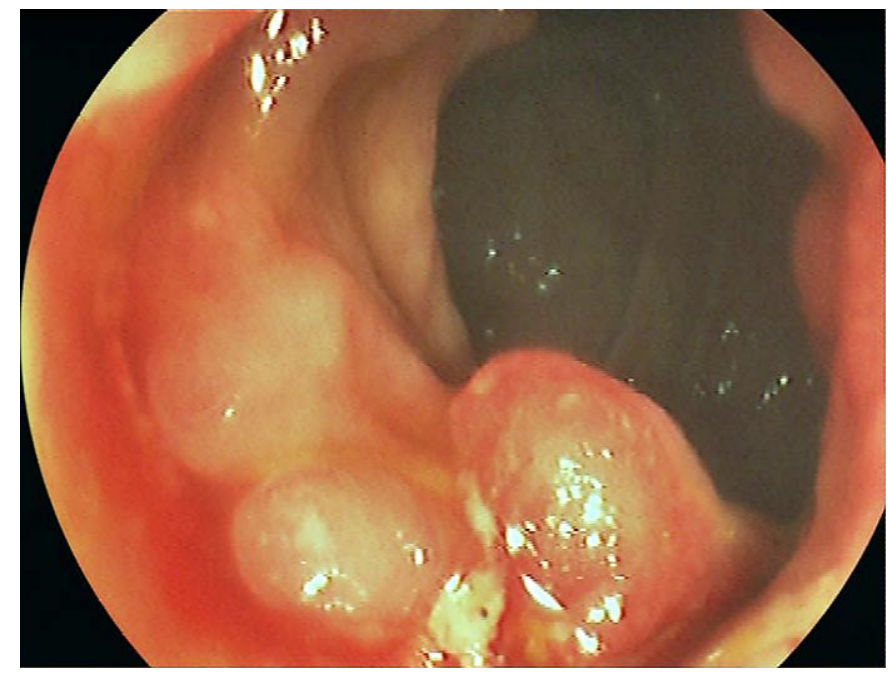

Figure 1 Colonoscopic view of a granuloma pyogenicum near the right flexure, showing a large friable tumor encircling the lumen.

\section{8}

In July 1999 we examined a 60-year-old woman who appeared to be in poor general health, with pale skin and lips. The only abnormality in the physical examination was found on palpation of the liver, which had a hardened consistency and was enlarged by about $6 \mathrm{~cm}$. Typical signs of liver cirrhosis were found on ultrasound examination and previously diagnosed esophageal varices were seen at gastroscopy. Colonoscopy revealed a large, vulnerable circular growing tumor near the right flexure; the tumor was only just passable by the colonoscope (Figure 1). A pseudopolypoid-like region was seen near the tumor. The remainder of the colon showed no abnormality. Histological examination of biopsy material revealed inflammatory and infiltrative granulation tissue.

Computed tomography showed only signs of liver cirrhosis: the tumor could not be seen and there were no enlarged lymph nodes. It must be remembered that the new techniques which would have been able to detect the lesion, such as virtual colonoscopy, were not available to us at this time [1].

The patient underwent operation because of the suspicion of colonic cancer and the presence of signs of obstruction. Intraop- erative examination by inspection and palpation seemed to confirm the diagnosis of colonic cancer and so an extended right hemicolectomy was performed. The postoperative course and recovery were uneventful. The histological diagnosis was of a granuloma pyogenicum of the colon.

Granuloma pyogenicum is a benign, angiomatous and vulnerable skin tumor, which often occurs a few weeks after an inflammatory traumatic condition. It can also arise spontaneously. In most cases it is a soft, ball-like, sessile tumor, with an incomplete epithelial layer. The surface is often friable and covered with encrusted blood. In most cases granuloma pyogenicum arises on the face, but the tumor may occur on the hand, the foot, or on the skin around the umbilicus. The pathogenesis is thought to be one of over-reactive growth of granulation tissue after a trivial injury. There are only a few descriptions of the tumor in the medical literature. One paper reported a case in which a granuloma pyogenicum of the skin developed after therapy with flashlamp-pumped pulsed dye laser [2]. We found only one description of intestinal granuloma pyogenicum in the medical literature, and in that case the lesion was located in the ileum [3].
It should be noted that it is difficult to differentiate this condition from other chronic granulomatous inflammatory lesions of the intestine, such as those of Crohn's disease. However, even if granuloma pyogenicum is a very uncommon lesion in the intestine, it should be included in the differential diagnosis of gastrointestinal tumors. Surgery is required in these patients because of the difficulty in differentiating granuloma pyogenicum from colonic cancer and because of the vulnerability of the lesion and its obstructive pattern of growth.

\section{Hocke, H. Bosseckert}

Department of Internal Medicine I, Friedrich Schiller University, Jena, Germany.

\section{References}

${ }^{1}$ Bond JH. Colon polyps and cancer. Endoscopy 2003; 35: 27-35

${ }^{2}$ Abd-el-Raheem TA, Hohenleutner U, Landthaler M. Granuloma pyogenicum as a complication of flashlamp-pumped pulsed dye laser. Dermatology 1994; 189: $283-285$

${ }^{3}$ Coulier B, Coppens JF, Mailleux P et al. A cause of intestinal intussusception in an adult. J Belge Radiol 1994; 77: 121 - 123

\section{Corresponding Author}

\section{Hocke, M.D.}

Department of Internal Medicine I, Friedrich Schiller University

Erlanger Allee 101

07740 Jena

Germany

Fax: $\quad+49-3641-939404$

E-mail: Michael.hocke@med.unijena.de 